\title{
CROSSLINGUISTIC REPOSITORY OF POLITICAL METAPHORS: STRUCTURE AND METHODOLOGICAL ISSUES
}

\author{
Inna V. Skrynnikova \\ Volgograd State University, Volgograd, Russia
}

\begin{abstract}
The article presents a critical review of numerous approaches to the analysis of metaphor in various scientific traditions. The focus is set on the interdisciplinary methods applied in the metaphor studies which resulted in corpora, databases of figurative language and analysis of repositories of metaphors in English, French, and German. The question of reliable methods and procedures of identification of metaphors in modern linguistics is discussed in the article. The author praises efficiency of a metaphor methodology that was verified in the University of California (Berkley, USA), and considers possibility of its application for studies on Russian political metaphors through their comparison with English. The metaphors of government are used for exemplification of explanatory potential of an integrative approach that unites the opportunities of combining bottom-up and topdown procedures of identifying and analyzing metaphors within a single repository. The paper presents a procedure of deep multi-level semantic analysis of metaphors and dwells on techniques of formalizing the conception of neural cascades as applied to the analysis of metaphors. The author uncovers the problems in constructing the cross-linguistic (English and Russian) repository of political discourse metaphors. The proposed approach enables to determine interrelated conceptual networks and present metaphors at different levels of specificity and complexity of a multimodal model of metaphorical cognition.

Key words: conceptual metaphor, frame, image-schema, inferences, repository, computational modeling of metaphor.

Citation. Skrynnikova I.V. Crosslinguistic Repository of Political Metaphors: Structure and Methodological Issues. Vestnik Volgogradskogo gosudarstvennogo universiteta. Seriya 2. Yazykoznanie [Science Journal of Volgograd State University. Linguistics], 2019, vol. 18, no. 2, pp. 128-146. (in Russian). DOI: https://doi.org/10.15688/ jvolsu2.2019.2.12
\end{abstract}

УДК $81 ’ 373.612 .2: 32$

Дата поступления статьи: 21.01.2019

ББК 81.055

Дата поступления статьи: 31.05.2019

\section{КРОССЛИНГВИСТИЧЕСКИЙ РЕПОЗИТОРИЙ ПОЛИТИЧЕСКИХ МЕТАФОР: СТРУКТУРА И ПРОБЛЕМЫ МЕТОДОЛОГИИ}

\author{
Инна Валериевна Скрынникова \\ Волгоградский государственный университет, г. Волгоград, Россия
}

\begin{abstract}
Аннотация. В статье представлен критический обзор подходов к исследованию метафоры в различных научных традициях. Показаны преимущества междисциплинарных методов, применяемых при анализе ме-

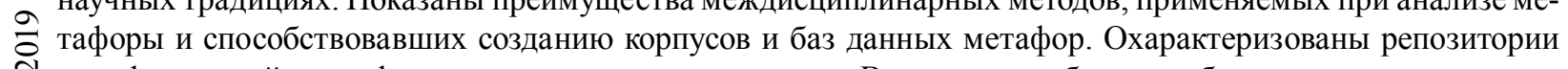
‥ метафор английского, французского и немецкого языков. Выявлены проблемы выбора надежных методов и процедур идентификации и анализа метафор в современной лингвистике. Описаны достоинства методолог гии анализа, апробированной в калифорнийском университете (Беркли, США), и обосновано ее применение до для анализа русских политических метафор в сопоставлении с английскими. На примере метафор управления продемонстрирован экспланаторный потенциал интегративного подхода, сочетающего нисходящие и восходящие методы идентификации и анализа метафор в рамках одного репозитория. В статье представлена процедура глубинного многоступенчатого семантического анализа метафоры и описана формальная реа() лизация концепции нейронных каскадов применительно к анализу метафор. Автором выявлены и классифи-
\end{abstract}


цированы проблемы, которые возникают в процессе создания кросслингвистического англо-русского репозитория метафор политического дискурса. Намечены перспективы предлагаемого интегративного подхода при определении взаимосвязанных концептуальных сетей, представлении метафоры на разных уровнях специфичности и сложности и создании мультимодальной модели метафорического мышления.

Ключевые слова: концептуальная метафора, фрейм, образ-схема, инференция, репозиторий, компьютерное моделирование метафоры.

Цитирование. Скрынникова И. В. Кросслингвистический репозиторий политических метафор: структура и проблемы методологии // Вестник Волгоградского государственного университета. Серия 2, Языкознание. -2019. - Т. 18, № 2. - С. 128-146. - DOI: https://doi.org/10.15688/jvolsu2.2019.2.12

\section{Введение}

Многочисленные исследования последних лет убедительно доказали, что метафора является когнитивным механизмом человеческого мышления и чаще всего используется носителями языка неосознанно. Она оказывает влияние на то, как люди размышляют о сложных реалиях, что приводит к формированию обычаев, ценностей, убеждений и идеологий. В разных языках наблюдаются как сходства в наборе первичных метафор, лежащих в основе процессов метафоризации, так и значительные несовпадения метафорических моделей на уровнях инференций и импликаций [Kövecses, 2010]. Этот факт делает метафору особенно перспективным объектом междисциплинарных исследований и свидетельствует о необходимости создания кросслингвистического репозитория метафор, отражающего области совпадений и различий в концептуализации сложных абстрактных сущностей носителями разных языков.

С учетом принципов, на которых базируются многочисленные теории метафоры (теории концептуальной метафоры [Lakoff, 1993; Lakoff, Johnson, 1980], модели концептуального наложения [Politics, Gender and Conceptual Metaphors, 2009], модели структурного наложения [Wolff, Gentner, 2000] и гипотезы категоризации атрибутов [McGlone, 1996]), были составлены и опубликованы словари и сборники метафор: словарь основных метафор Master Metaphor List [Lakoff, 1994], в котором метафоры английского языка группируются в соответствии с их концептуальным наложением; гамбургская база метафор французского и немецкого языков (HMD) [Eilts, Lönneker, 2002]; EuroWordNet, где представлены синсеты на основе сфер-источников и сфер-мишеней словаря метафор (MML), которые указы- вают на определенные источники метафорической семантики во французском и немецком языках. На разработку инструментов выявления и распознавания образного языка направляют свои усилия специалисты в области вычислительной лингвистики [Bogdanova, 2010; Li, Sporleder, 2010; Peters, Wilks, 2003; Shutova, 2011], где одним из аспектов исследований стала идентификация метафорических выражений в тексте [Fass, 1991]. Проводится также кластеризация имен и глаголов [Shutova, Sun, Korhonen, 2010], которая начинается с небольшого набора образцов однословных метафор и заканчивается кластерами, представляющими концепты сферы-источника и сферы-мишени и связанными определенными метафорическими отношениями. Эти кластеры затем используются для аннотирования метафоричности текста.

Однако в упомянутых и других публикациях вне поля исследовательского внимания остаются некоторые важные аспекты анализа метафоры: описание грамматических конструкций, содержащих метафоры, с учетом различий в синтаксическом строе неблизкородственных языков, выявление конвенциональности (стертости) или новизны метафоры, ее неосознанного / преднамеренного использования носителями языка, влияния контекста и типа дискурса на ее интерпретацию.

\section{Обзор существующих ресурсов метафор}

После так называемого когнитивного поворота в лингвистике и создания теории концептуальной метафоры (ТКМ) в 1980-х годах, многочисленные исследования опирались на ее основные положения и принципы. Революционная идея о том, что метафора служит не только лингвистическим орнаментом, но и является когнитивным механизмом мышле- 
ния, побудила ученых проводить анализ концептуальных метафорических моделей в разных типах дискурса как на материале одного языка, так и в сопоставительном аспекте. Несмотря на беспрецедентную популярность ТКМ, представленной в книге «Метафоры, которыми мы живем» [Lakoff, Johnson, 1980; 2003], некоторые исследователи позднее поставили под сомнение обоснованность методов, применяемых в ней и доказывающих когнитивную природу метафоры [Deignan, 2010; Gibbs, 2007, p. 7; 2011, p. 533-537; Low, Todd, 2010; Steen et al., 2010a, 2010b]. Основная критика касается того, что большинство примеров, приводимых Дж. Лакоффом и М. Джонсоном, взяты не из естественно возникающего дискурса и, следовательно, являются искусственными. Чрезмерное внимание к концептуальному характеру функционирования метафоры привело к многочисленным исследованиям, в которых нет сложных процедур сбора лингвистических данных и идентификации метафоры. Так, Л. Камерон считает, что акцент на когнитивных аспектах привел к необоснованному отсутствию интереса к языку метафоры [Researching and applying metaphor..., 1999, p. 11].

Идентификация и аннотация метафоры представляют собой сложные процедуры, что обусловлено несколькими факторами. Во-первых, метафора в языке проявляется разнообразными способами и может быть обнаружена как в случайных, новых выражениях, так и в конвенциональных выражениях, метафорический потенциал которых едва ли идентифицируется. Тот факт, что метафоричность градуальна [Hanks, 2006], послужил основанием для разработки различных подходов к метафоре и расходящихся взглядов на то, что считается метафорой. Во-вторых, несмотря на большое количество исследований по метафоре, среди ученых до сих пор нет единодушия относительно достоверных и надежных методов и принципов идентификации метафоры в языке. Поэтому специалисты в течение многих лет в основном полагались на свою интуицию, определяя метафоричность слова или фразы в (кон)тексте. Однако первазивный характер метафоры привел к многочисленным попыткам создания баз, репозиториев и банков метафор как для одного языка, так и для нескольких языков.

Одной из первых попыток систематизации и классификации метафор было составление перечня базовых метафор, выполненное коллективом лингвистов-когнитивистов (G. Lakoff, J. Espenson, A. Goldberg) в Калифорнийском университете, Беркли (The University of California, Berkeley) в 1989 г. [Lakoff, 1994] на основе книг «The Conduit Metaphor» M. Редди [Reddy, 1979] и «Metaphors we live by» Дж. Лакоффа и М. Джонсона [Lakoff, Johnson, 1980], а также студенческих работ. В этом перечне метафоры зафиксированы и проанализированы в едином формате в соответствии с группой, которую они представляли (например, метафоры структуры событий, эмоций, ментальных событий и т. д.).

Реализаций другого подхода к идентификации и анализу метафоры стала база данных Metalude, разработанная на факультете английского языка в университете Линнан (Гонконг). Это интерактивная база данных конвенциональных лексикализованных метафор, представляющих английские и китайские метафорические лексические элементы с буквальными и метафорическими значениями, грамматическими особенностями и примерами их использования. Языковые метафоры в Metalude сгруппированы в соответствии с типом концептуальных метафор, которые они вербализуют, или с учетом корневых аналогий, то есть метафорических паттернов, которые имеют решающее значение для носителей обоих языков [Goatly, 2007]. В отличие от стандартного метафорического списка Беркли и тезауруса традиционных английских метафор [Wilkinson, 2002], организованного в соответствии со сферой-источником, Metalude опирается на лексикографический подход к анализу метафоры. Для включения в базу лексические единицы проверяются на наличие определенных метафорических значений в словаре современного английского языка. Корневые аналогии фиксируются, если общее количество лексических элементов, реализующих корневую аналогию, не меньше, чем шесть, а количество токенов этих лексических элементов в блоке Cobuild Bank of English / Words Online составляет не менее 200. Список основных метафор Дж. Лакоффа и карта 
корневых аналогий Э. Гоэтли - это два ресурса, которые устанавливают взаимосвязь между метафорами на основе импликаций.

В последние годы динамично развивающиеся корпусные исследования позволили разработать и применять более сложные процедуры идентификации метафор. В настоящее время идентификация метафоры, как правило, осуществляется на основе анализа в двух разных, хотя и не взаимоисключающих, направлениях: сверху вниз (top-down) и снизу вверх (bottom-up). Анализ «сверху вниз» состоит в установлении некоторого набора концептуальных метафор и дальнейшем извлечении поверхностных лингвистических метафор из текста, анализ «снизу вверх» - в выявлении концептуальных соответствий из лингвистических выражений, помеченных как метафорически используемые, и дальнейшем определении концептуальных метафор.

Методологию идентификации метафоры в корпусной лингвистике можно отнести к подходу «сверху вниз». Однако исследователи метафоры стали осознавать ограниченность этого метода и отдавать предпочтение подход «снизу вверх». Все больше подчеркиваются различия между грамматикой и конкретным использованием языка [Steen, 2007], что приводит к изучению метафоры в конкретных контекстах и усложнению задачи ее идентификации [Krennmayr, 2013].

Группой лингвистов, известной как Pragglejaz Group (2007), был апробирован метод идентификации метафоры (Metaphor Identification Procedure - MIP) для выявления частотности метафорически используемых слов. Основная проблема в МIP - это определение соотношения основного и контекстуального значений лексических единиц [Steen et al., 2010a, p. 35]. MIPVU (Metaphor Identification Procedure Vrije Universiteit), разработанная пятью годами позже, служит более надежным инструментом для идентификации метафорического потенциала слов в дискурсе как проявлений базовых междоменных соответствий, хотя последние не являются основным объектом анализа MIPVU.

Недавнее амбициозное начинание в области кросслингвистического анализа метафор проект MetaNet (https://metaphor.icsi.berkeley.edu/ pub/en/), реализующийся в Международном ин- ституте компьютерных наук, Беркли (International Computer Science Institute, Berkeley) и объединяющий специалистов в области когнитивной лингвистики, психологии, нейронауки и информатики. Этот коллектив разработал инновационный инструмент формализации теории концептуальной метафоры и ее реализации в структурированном репозитории метафоры [Stickels et al., 2016]. В основе анализа лежит как внутренняя структура фреймов, так и отношения между ними, основанные на принципах грамматики воплощенных конструкций (Embodied Construction Grammar). Эта грамматика «подпитывает» структуру метафор и отношения между ними. Очевидным преимуществом подхода MetaNet к созданию метафорического репозитория является открываемая им возможность представить иерархический характер отношений межу метафорами и фреймами, причем информация о возникающих инференциях сохраняется во всей сети. Различия между языками, обнаруженные в высказываниях из естественно возникающего дискурса, могут быть уточнены, что позволит существенно расширить анализ метафоры. Несмотря на неоспоримую систематичность в наборах связанных между собой метафорических выражений, сущность и характер такой систематичности требует дальнейшей разработки. При создании многоязычных репозиториев метафор эта проблема становится еще более актуальной как для определения культурно-специфичных типов отношений, так и для поиска решений этой задачи методами вычислительной лингвистики.

\section{Опыт создания двуязычного репозитория политических метафор: новизна, задачи и методы}

Принимая во внимание систему отношений между метафорами (базовыми и сложными) и опираясь на методологию глубокого четырехступенчатого семантического анализа метафор, апробированную в проекте MetaNet (основные достоинства которого описаны выше), автором статьи предпринята попытка интегрировать методы компьютерного моделирования, нейронной теории языка (метафорических каскадов) [Lakoff, 2008] и 
критического анализа дискурса в ходе создания двуязычного репозитория политических метафор. Цель данной статьи состоит в описании процедуры анализа политических метафор русского и английского языков на основе системы «semantic media-Wiki» (программного обеспечения, позволяющего добавлять семантические аннотации, используя дополнительные элементы разметки). К достоинствам создаваемого репозитория относится то, что он не просто представляет список метафор в русском и английском языках, но является онтологией метафорических «семей» со всеми присущими им отношениями, возникающими между образ-схемами, фреймами, отражая междоменные соответствия разного уровня. Отношения между метафорами и фреймами автоматически фиксируются графически. Основу корпуса русских метафор составляют примеры из словаря русских политических метафор (А. Баранов, Ю. Караулов, 1994), Национального корпуса русского языка. Репозиторий постоянно пополняется новыми метафорами, извлекаемыми методом сплошной выборки из различных текстовых источников (журнальные статьи, официальные правительственные сайты, политические блоги, социальные сети) на английском и русском языках. При создании репозитория реализована возможность (полу)автоматического извлечения языковых метафор из текстов на основе шаблонов, которые были введены вручную. Поскольку для метафор русского языка все еще отсутствуют репозитории, аналогичные FrameNet и MetaNet, создание такого хранилища закладывает основы для дальнейшего применения полученных данных в лингвистических исследованиях.

Основная цель создания кросслингвистического репозитория метафор заключается в том, чтобы определить, как метафоры отражают языковую, концептуальную и культурную среду, в которой они развивались.

Конечные цели построения репозитория:

- создать первоначально двуязычную (англо-русскую) поисковую базу данных политических метафор с перспективой расширения спектра языков;

- выделить взаимосвязанные концептуальные сети, созданные каскадной теорией применительно к политическому дискурсу;
- представить метафоры на разных уровнях их специфичности и сложности (базовые, вторичные, сопряженные);

- выявить области пересечения метафорических моделей в анализируемых языках, а также специфичные для отдельной культуры модели концептуализации политических процессов носителями соответствующих языков, раскрывающие области гиперкогниции;

- создать репозиторий, отражающий функционирование метафор различного уровня в разных модальностях (языковой, визуальной, жестовой) и существующие отношения между ними.

На примере исследования политических метафор и их реализации в английском и русском языках показано, каким образом качественные и количественные методы анализа могут сочетаться в одной базе данных.

Новизна предлагаемой процедуры создания кросслингвистического (русско-английского) репозитория политических метафор заключается во внедрении и формальной реализации концепции метафорических каскадов применительно к глубокому семантическому анализу метафор. Каскад представляет собой иерархически организованную концептуальную комбинацию образ-схем, фреймов и метафор, которые употребляются носителями языка достаточно часто, чтобы быть зафиксированными как единая сложная концептуальная сущность. При этом каждая из частей каскада продолжает функционировать отдельно. Специфические новые языковые метафоры обычно используют уже существующие высокоуровневые структуры. Понятие каскада основывается на изучении данных фреймовой семантики, согласно которым фреймы представляют собой группу (набор) когерентных ролей, динамически связанных друг с другом [Fillmore, 1976; Ruppenhofer, Brandes, 2016], а в теории концептуальной метафоры, как и в нейронной теории языка в целом, метафоры являются по существу пучками концептуальных соответствий, образуемых фреймами внутри доменов (сфер) [Lakoff, 1993].

На нейронном уровне каскадообразная схема представляет собой полученную на основе накопленного опыта сеть, которая объединяет воплощенную (embodied) систему межнейронных связей в разных областях 
мозга [Lakoff, 2008]. Каскад отражает межнейронные связи, которые, когда активны, (1) связывают локализованные схемы в области мозга на уровне нейронов и (2) создают интегрированное целое из сложных частей. Далее каскадная схема может применяться и повторно использоваться при обработке мысли и языка. Система межнейронных связей лежит в основе образ-схем, устойчивой образности, кластеров концептуальной интеграции [Fauconnier, Turner, 1998] и метафорических концептуальных наложений. Ее важнейшими частями являются первичные воплощенные образ-схемы и метафоры, потому что информация из нейронных цепей, непосредственно связанных с телом (нейронные сети двигательных актов, восприятия, эмоций и мышления), обязательно активируется при использовании и понимании языка.

Методологический подход, применяемый в настоящем исследовании, сочетает в себе две теоретические традиции, обе из которых трактуют метафору как широко распространенную форму организации концептуальной структуры: нейронную теорию языка и метафоры [Feldman, 2006; Lakoff, 2008] и критический анализ дискурса [Charteris-Black, 2004]. Первая является наиболее перспективным направлением в когнитивной лингвистике, вторая представляет собой критический анализ метафор как более частный случай критического анализа дискурса. Сочетание этих двух методологических подходов к идентификации метафоры уже апробировано в на-

(1) языковая метафора

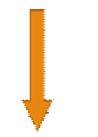

(2) концептуальная метафора

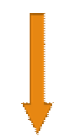

(3) сопряженная метафора

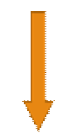

(4) метафорическая инференция уке (см., например: [Charteris-Black, 2004; Goatly, 2007; Cienki, 2008]), интеграция этих подходов при изучении политического дискурса также может привести к плодотворным результатам, стимулирующим дальнейшие разработки в этой области. Комбинация вышеупомянутых подходов позволяет, с одной стороны, опираться на методологический аппарат нейронной теории метафоры (определение концептуальной метафоры, теории доменов (сфер), концептуальных соответствий, образ-схем, фреймов, инференций и т. д.), а с другой стороны, идти по пути признания значимости метафор в построении социальной реальности вокруг нас [Musolff, 2004]. Таким образом, применяемая комплексная методология позволяет «примирить» подходы к идентификации метафоры «снизу вверх» и «сверху вниз» [Skrynnikova, 2016] с критическим анализом дискурса, при котором постулируется решающая роль метафоры в понимании социальной и культурной среды вокруг нас. Модель идентификации и комплексного анализа метафоры представлена на рисунке 1.

Ключевыми для понимания модели анализа метафоры в репозитории являются фундаментальные положения когнитивной семантики. Основной объект анализа, фрейм [Fillmore, 1976; Lakoff, 1987; Langacker, 2008] включает в себя когнитивные структуры, поддерживающие основные семантические концепты (примитивы). Это ведет нас к пониманию фрейма как четко структурированной единицы, которая является схематическим
(3) классификация метафор в языке как

«поверхностной» реализации концептуальной

метафоры

(2) построение концептуального пространства

каждой сферы-мишени

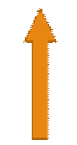

(1) сбор данных о потенциальной

сфере-мишени из корпусов

Рис. 1. Модель идентификации и анализа политической метафоры в репозитории 
представлением наших знаний о мире. Таксономические отношения между фреймами устанавливаются путем определения их универсальности и специфичности, которые становятся очевидными, когда те или иные более конкретные фреймы создают более общие [Clausner, Croft, 1999]. Анализ фреймов английского языка частично импортирован в репозиторий из MetaNet Metaphor Wiki (pecypc доступен в Интернете), где некоторые фреймы в свою очередь базируются непосредственно на электронной базе фреймов FrameNet, остальные анализируются и вводятся в репозиторий вручную. Поскольку для русского языка такие ресурсы, как FrameNet, до сих пор отсутствуют, анализ фреймов на начальной стадии базируется на синтаксически аннотированном корпусе Treebank.

\section{Метафора как инструмент убеждения}

Изучение метафоры как мощного механизма, оказывающего воздействие на взгляды людей относительно насущных социальных проблем, уже давно занимает центральное место в анализе политического дискурса [Будаев, Чудинов, 2018; Lakoff, 1996/2002; Politics, Gender and Conceptual Metaphors, 2009]. Heкоторые исследователи применяют концептуальную теорию метафоры, чтобы продемонстрировать ее значимость в построении и понимании политического дискурса [Musolff, 2004], который создается и разворачивается в результате экспликации концептов, отражающих мировоззрение тех, кто управляет, и тех, кем управляют. Современные ученые сосредоточены на анализе способов донесения до социума сложных идей посредством метафор как инструментов манипулирования общественным мнением и разрешения конфликта между культурной самобытностью и общечеловеческими ценностями. Примечательно, что толкование одного и того же концепта и вытекающих из него инференций может различаться как в рамках одной культуры, так и в разных культурах [Болдырев, 2013]. Образный язык в целом и метафора в дискурсе в частности имеют как лингвистическое, так и концептуальное наполнение и являются инструментами фрейминга [Burgers, Konijn, Steen, 2016, p. 412]. Убеждающая сила метафор нео- днократно подтверждалась мета-анализом, раскрывающим силу и эффективность метафоры в воздействии на аудиторию (см., например: [Sopory, Dillard, 2002]).

Многочисленные исследования последних десятилетий наглядно продемонстрировали потенциал убеждения метафорического языка, а роль метафоры как инструмента, влияющего на общественное мнение, и мощного средства манипулирования взглядами людей, едва ли вызывает сомнение. Модели употребления метафорического языка и превалирование определенных метафорических моделей, используемых государственными субъектами (руководящими органами) и управляемыми субъектами, могут не только дать представление о культуре, но и показать сходства и различия в их взглядах на возможные способы решения таких критических проблем, как контроль над оружием, права человека, демократия, коррупция, аборты и т. д.

Все упомянутое выше доказывает целесообразность изучения метафор и дальнейшей систематизации наших знаний о роли метафор в политическом дискурсе, обосновывая необходимость построения репозитория метафор, который послужит базой для будущих разысканий.

\section{Метафоры управления в репозитории}

Проведение кросслингвистического исследования метафор в дискурсе предполагает сравнение и противопоставление присущих каждому языку метафорических моделей, а также анализ и представление целостной картины основных культурных особенностей, сравнение и противопоставление моральных взглядов, влияющих на предпочтительные способы концептуализации политических процессов.

В рамках данной статьи процедура извлечения и анализа метафоры демонстрируется на примере одного из кластеров политических метафор, метафор управления (governance metaphors), отобранных из различных текстовых источников и корпусов американских и российских СМИ. Глубинный семантический анализ этого кластера метафор в репозитории позволяет выявить на инференциальном уровне то, как метафоры отражают 
культурные и идеологические вариации в концептуализации управления, приводящие к трудностям их понимания носителями других языков. Каждая такая метафора выражает концепт сферы-мишени в терминах концепта сферы-источника. В качестве иллюстрации возможностей интегрирующего подхода к анализу метафоры рассмотрим высказывание президента России В.В. Путина во время прямой линии в июне 2018 г., выражающее его позицию в связи с провокационными действиями стран Запада: Иногда я думаю, может быть, лучше, чтобы наш медведь сидел тихо, а не гонялся по лесу за поросятами. В нем можно выделить сферу-источник (животные), и сферу-мишень (политики или на более высоком уровне - управленческие структуры). Концептуальное соответствие (mapping) между сферой-источником и сфероймишенью и является концептуальной метафорой, которая реализуется на языковом уровне совокупностью лингвистических метафор. Схема анализа представлена на рисунке 2 .

Четырехступенчатый анализ метафоры позволяет выявлять существующие импликации и инференции. Однако при такой процедуре анализа метафор, ограниченной определенным набором шаблонов анализа в media-Wiki, не фиксируются характеристики, приписываемые конкретному типу животного в той или иной лингвокультуре. Таким образом, не находит отражения культурная информация, закодированная в конкретной метафоре, что свидетельствует о сложности исчерпывающего анализа метафор в дискурсе.

Иллюстрируя процесс создания репозитория политических метафор и характеризуя возникающие в связи с этим проблемы, в статье мы ограничимся одной сферой-мишенью - управление. Нами определен набор потенциальных сфер-источников, начиная со сферы-источника первичных метафор, которые предположительно являются универсальными. Затем этот набор был расширен за счет включения сфер-источников, которые упоминаются в научной литературе о метафоре, а также те примеры метафор управления, которые были извлечены автором статьи из разных текстовых источников и внесены в репозиторий вручную (всего анализу подвергнуто 252 метафоры).

\section{Методологические проблемы составления репозитория политических метафор}

Исследователи утверждают, что изучение языковых особенностей, а также когнитивных и коммуникативных аспектов метафоры является фундаментальной предпосылкой
(1) языковая метафора

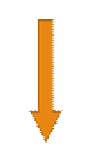

(2) концептуальная метафора

(3) сопряженная метафора

(4) метафорическая инференция
Может быть, лучше, чтобы наш медведь сидел тихо, а не гонялся по лесу за поросятами

пОЛИТИКИ $\leftarrow$ жИВОТНЫЕ

ОППОЗИЦИОННЫЕ ПОЛИТИКИ $\leftarrow$ ЖИВОТНЫЕ, ПРЕСЛЕДУЮЩИЕ ДРУГ ДРУГА

\author{
СТРАНА $\leftarrow$ ЛЕС \\ ЗАЩИТА НАЦИОНАЛЬНЫХ ГРАНИЦ $\leftarrow$ ИЗГНАНИЕ \\ ВРАЖДЕБНЫХ ЖИВОТНЫХ ИЗ ЛЕСА
}

Терпимость к присутствию враждебных животных вблизи от леса может нарушить его нормальную жизнедеятельность и нанести вред или уничтожить животных, мирно обитающих в нем. Защита леса означает сохранение его целостности путем предотвращения доступа к нему враждебных животных.

Рис. 2. Схема глубинного семантического анализа метафор 
понимания ее природы и способов воздействия на способы мышления людей [Gibbs, 2008; Researching and applying metaphor..., 1999]. Решение исследовательских задач построения репозитория неизбежно приводит к возникновению методологических проблем, связанных с возможностью адекватного отражения всех этих аспектов в одном репозитории. Эти проблемы становятся особенно очевидными, когда лингвисты занимаются сопоставлением метафорических данных в таких неблизкородственных языках (например, английском и русском).

Решение задачи построения репозитория метафор политического дискурса предполагает не только анализ универсальных и специфичных для сопоставляемых языков способов метафорической концептуализации, но и изучение культурного и идеологического подтекста, затрагивающего определенные модели концептуализации. Проблемы, возникающие в ходе создания репозитория, можно разделить на проблемы, имеющие лингвокогнитивный характер, и проблемы, связанные с вычислительной стороной проекта, то есть обусловленные сложностью разработки электронной оболочки репозитория.

\section{Проблемы \\ лингвокогнитивного характера}

Одна из первых проблем, которая возникает в процессе создания репозитория, - это выбор типа конструкции, принятого для метафорического анализа в обоих языках из-за различий в их структуре. Метафоры различаются в зависимости от того, как они выражаются грамматически в языке. Сфера-источник и сфера-мишень накладывают ограничения на набор синтаксических ролей, которые могут быть отражены в метафоре, и, следовательно, на синтаксические варианты выражения метафорического значения. В русском языке свободный порядок слов может способствовать большему количеству синтаксических конструкций, содержащих метафору. Задача исследователя состоит в том, чтобы представить эту информацию в репозитории, сравнивая языки различного синтаксического строя. Другая проблема заключается в поиске способа установления синтаксических конструкций, используемых для передачи метафорических значений, в определении уровня, на котором необходимо выполнять аннотирование.

Поскольку политические метафоры рассматриваются на уровне дискурса, их можно назвать расширенными (распространенными) метафорами, представляющими непрерывный сценарий. Следовательно, определенный нарратив создается только в результате взаимодействия нескольких метафор одновременно, что невозможно отразить в репозитории с ограниченным набором темплейтов для анализа. Примером такой метафоры является метафора европейской интеграции:

ЕВРОИНТЕГРАЦИЯ (ПОЛИТИЧЕСКИЕ ПРОЦЕССЫ) $\leftarrow$ ПУТЕШЕСТВИЕ

СТРАНЫ ЕС $\leftarrow$ ВАГОНЫ ПОЕЗДА

НАРОДЫ СТРАНЕС $\leftarrow$ ПАССАЖИРЫ СООТВЕТСТВУЮЩИХ ВАГОНОВ

ЦЕЛИ $\leftarrow$ (НЕИЗВЕСТНЫЕ) ПУНКТЫ НАЗНАЧЕНИЯ

Не менее важна проблема того, что опыт взаимодействия человека с окружающей действительностью, лежащий в основе якобы универсальных метафор (например, метафоры гнева), может варьироваться в разных лингвокультурах. Следовательно, во многих случаях универсальность опыта нашего телесного взаимодействия с окружающим миром не обязательно приводит к универсальной концептуализации - по крайней мере, не на уровне вторичных метафор, которые могут возникнуть из совокупности нескольких первичных метафор. Различия в наборе предпочтительных метафор, используемых политическими субъектами в определенной культуре, также могут быть результатом истории общества и личной истории [Kövecses, 2010]. По Дж. Лакоффу, американская политика структурирована двумя противоположными воззрениями на руководителя, основанными на двух различных семейных моделях: Строгий отец (Strict Father) и Заботливый родитель (Nurturant Parent) [Lakoff, 2010], отражающих разные моральные взгляды на семью республиканцев и демократов соответственно. Нами предложена схема репрезентации модели моральных суждений по Дж. Лакоффу (рис. 3). 
Однако эта модель едва ли может быть применена к российскому политическому дискурсу ввиду отсутствия четко определенной дихотомии между консерваторами и либералами и незрелости парламентской системы в России.

Глубинный анализ метафоры НАЦИЯ - это СЕМЬЯ, которая, на первый взгляд, универсальна для русской и английской лингвокультур, показывает, что различные инференции и сопряженные метафоры результат применения разных моделей морали. Российский политический дискурс, как показывает проанализированный нами языковой материал, во многом строится на модели «руководитель - добрый и мудрый царь-отец». В какой-то степени эта модель является «пограничным» случаем между «Строгим отцом» и «Заботливым родителем». С одной стороны, добрый и мудрый царь-отец неограничен во власти над своими детьми, отдавая приказы, наказывая тех, кто не подчиняется, и даря подарки послушным детям. Однако, поскольку у отца много детей (региональных губернаторов), он не всегда может контролировать их действия, что приводит к возможным проступкам или аморальному поведению (бесхозяйственность, бюрократия, коррупция, и т. д.). Борьба с последней, будучи важной частью государственного управления, получила отра- жение в языке и стала источником разнообразной метафорической концептуализации. Примеры анализа англо- и русскоязычных метафор в репозитории представлены ниже с указанием междоменных соответствий на рисунке 4.

В репозитории зафиксированы также другие универсальные метафоры: КОРРУПЦИЯ - это ЭРОЗИЯ, КОРРУПЦИЯ - это ДИКИЙ ЗВЕРЬ, КОРРУПЦИЯ - эТО РАСТЕНИЕ (СОРНЯК), КОРРУПЦИЯ - этО ГРЯЗЬ.

Семантический анализ метафор управления (464 единицы, из которых 252 русских и 212 английских) позволил сделать вывод о том, что в целом носители русского и английского языков разделяют отрицательное отношение к коррупции как к проявлению безнравственного поведения. Это подтверждают сходные метафорические модели в обоих языках. Однако, несмотря на значительное сходство в концептуализации коррупции в разных языках, были обнаружены специфические для русского языка метафорические модели (например, КОРРУПЦИЯ - СТИХИЙНОЕ БЕДСТВИЕ, КОРРУПЦИЯ - НЕПРЕОДОЛИМАЯ СИЛА). Интересным представляется пример специфичной концептуализации коррупции как природного бедствия, уникальность которой проявляется на уровне анализа инференций (рис. 5).

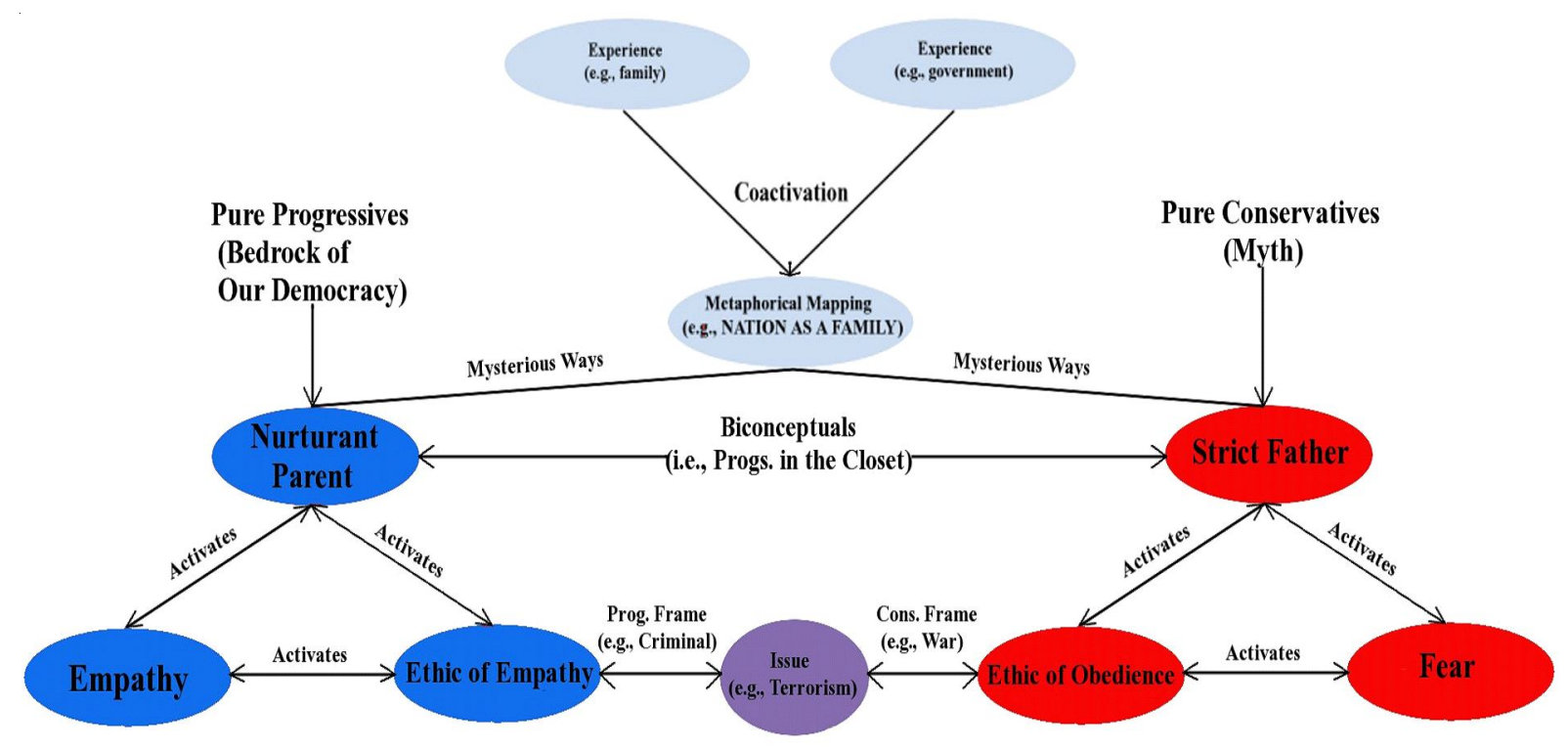

Рис. 3. Графическая репрезентация модели моральных суждений 


\section{МЕЖКУЛЬТУРНАЯ КОММУНИКАЦИЯ}

(1) языковая метафора

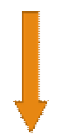

(2) концептуальная метафора

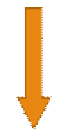

(3) сопряженная метафора

(4) метафорическая инференция
The 2015 Corruption Perceptions Index shows that corruption remains a blight.

Коррупция на протяжении веков является раковой опухолью на теле России.

КОРРУПЦИЯ $\leftarrow$ БОЛЕЗНЬ (ВИРУС)

КОРРУПЦИЯ ६ ИНФЕКЦИЯ (ВИРУС)

ЧИНОВНИКИ ПРАВИТЕЛЬСТВА $\leftarrow$ ИНФИЦИРОВАННОЕ ТЕЛО

УЩЕРБ ОТ КОРРУПЦИИ В ПРАВИТЕЛЬСТВЕ $\leftarrow$ НАРУШЕНИЕ

ЗДОРОВЬЯ НАЦИИ

Инфекцию необх одимо лечить

Инфицированные должны быть изолированы от других, чтобы предотвратить распространение вируса.

Здоровые должны быть привиты от инфекции.

Существующей вакцины недостаточно, чтобы привить всех.

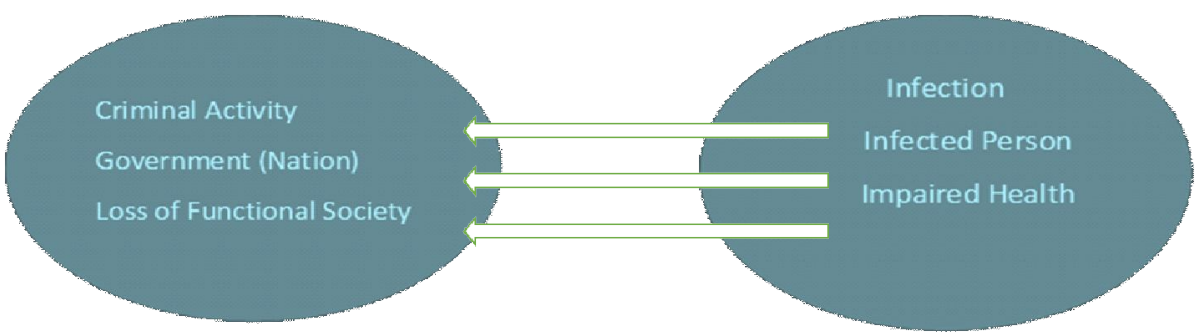

Рис. 4. Междоменные соответствия метафоры КОРРУПЦИЯ $\leftarrow$ ВИРУС (CRIMINALACTIVITY is a VIRUS)

(1) языковая метафора

(2) концептуальная метафора

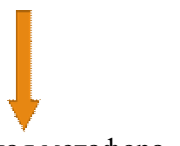

(3) сопряженная метафора

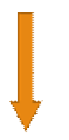

(4) метафорическая инференция
Коррупиия - это ураган, сметаюший все на своем пути. Коррупиия бушует в правоохранительных органах. Взяточничество уносит деньги людей.

РОССИЙСКАЯ КОРРУПЦИЯ $\leftarrow$ ПРИРОДНОЕ БЕДСТВИЕ

КОРРУПЦИЯ ६ НЕКОНТРОЛИРУЕМАЯ ПРИРОДНАЯ СИЛА

НАЦИЯ Ł НЕЗАЩИЩЕННАЯ СУЩНОСТЬ

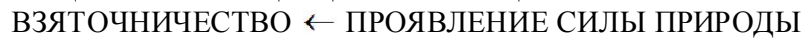

ТЕРПИМОСТЬ К КОРРУПЦИИ $\leftarrow$ ОБРЕЧЕННОСТЬ ПЕРЕД

СИЛАМИ ПРИРОДЫ

Природные бедствия - это действия Бога.

Божью волю следует терпеть и принимать на благо лучшего будущего.

Природные бедствия не могут быть предотвращены.

Рис. 5. Схема глубинного семантического анализа метафоры КОРРУПЦИЯ $\leftarrow$ СТИХИЙНОЕ БЕДСТВИЕ 
Такая метафора является более частным случаем базовой метафоры СОБЫТИЯ - это СИЛЫ. Эта модель подчеркивает непредсказуемость, быстроту и беспомощность людей, пострадавших от бедствия. Инференция отражает укоренившиеся убеждения и суеверия русского народа, связанные с природной силой (бедствием), которые есть отражение как архаичного языческого, так и православного мировосприятия. Языческое мировосприятие определяет понимание стихийного бедствия как наказания людей, которые не угодили духам, православное мировоззрение обусловливает понимание событий, происходящих в жизни людей, как проявление воли Бога, а следовательно, они должны восприниматься со смирением.

Наличие в русской лингвокультуре специфических метафор, концептуализирующих коррупцию, дает основания классифицировать концепт «Коррупция» как спорный концепт (contested concept) (термин Дж. Лакоффа).

Приведенные выше примеры свидетельствуют о сложности адекватного сравнительного изучения моделей морали, лежащих в основе потенциально различных способов концептуализации одних и тех же объектов, основанных на разных культурных традициях. Следовательно, в силу несоответствия между моделями морали их носителей возникает проблема противопоставления метафор государственного управления в двух языках. Представляется сложным также графически отображать в репозитории области гиперкогниции, хотя это является одной из важных задач его построения. На рисунке 6 представлен фрагмент иерархически организованной модели отношений метафор морали в английском языке с указанием всех типов отношений между метафорами разных уровней. Предложить аналогичную модель для русского языка на данном этапе создания репозитория пока невозможно ввиду отсутствия русскоязычной версии FrameNet.

Кроме рассмотренных проблем, в ходе создания репозитория неизбежно возникают сопутствующие им вопросы: а) какие сегменты языка следует привлечь для анализа и сравнения; б) каковы процедуры сравнения и сопоставления метафор как на лингвистическом, концептуальном, так и на дискурсивном уровне; в) каковы способы выделения тонких различий, выявленных на уровне инференций, особенно для кажущихся универсальными метафор; г) каковы критерии идентификации универсальности и вариативности метафор. Ответы на эти вопросы существенно расширят возможности применения интегративного подхода к анализу метафор и отражения в репозитории сложных отношений разного уровня между ними.

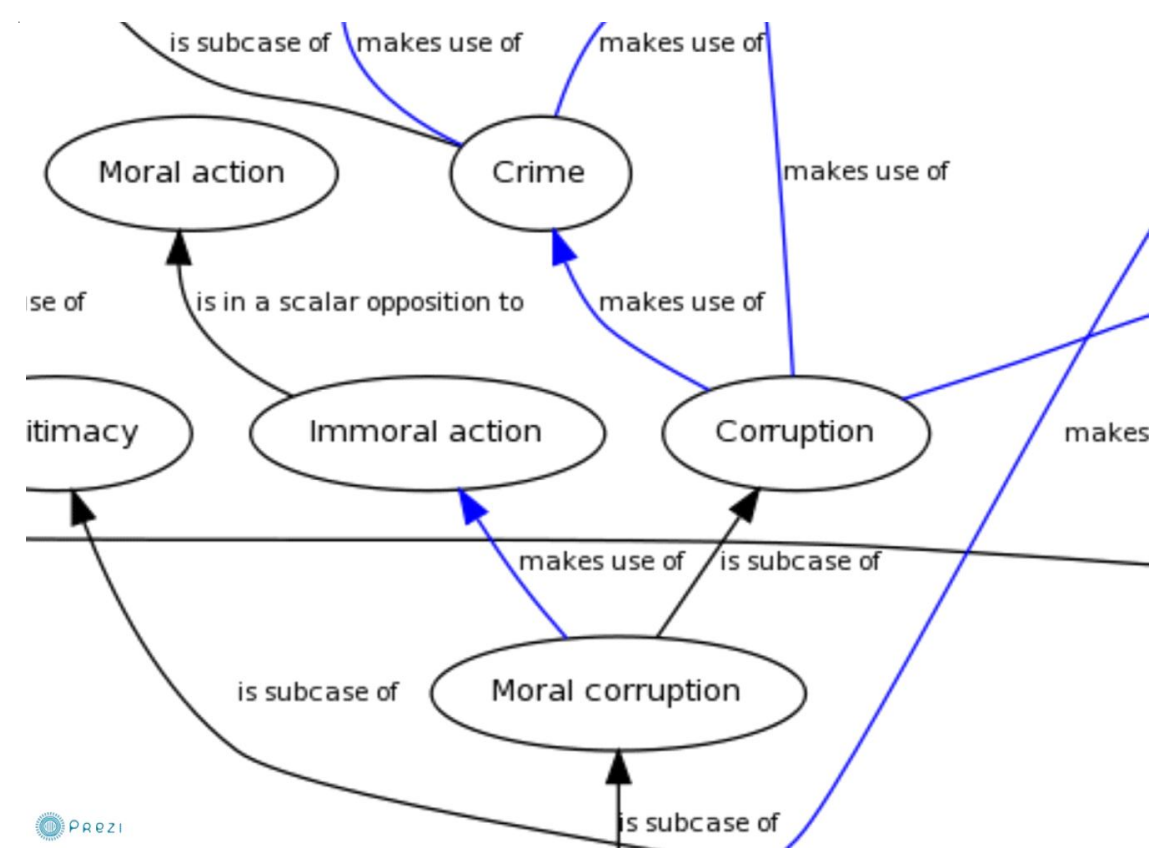

Рис. 6. Иерархическая модель отношений метафор морали в английском языке (источник: MetaNet) 


\section{Проблемы \\ компьютерного моделирования метафоры}

Специалисты в области вычислительной лингвистики, участвующие в исследованиях метафоры, обычно полагаются на ранее аннотированные наборы данных. Однако создание таких баз данных метафор является сложной задачей. Сложность объясняется тем, что проведение любого семантически разнообразного аннотирования - это начинание, которое требует строго определенного стандарта аннотации, согласованного между участвующими в нем учеными. Даже высококвалифицированные аннотаторы, использующие специализированные инструменты, склонны не соглашаться относительно смысла отдельных предложений [Banarescu et al., 2013]. В предпринятых ранее попытках разметки метафор трудности возникали даже при аннотировании метафоры с метками сферы-источника и сферы-мишени, проводимом вручную [Shutova, Sun, 2013], что обусловлено отсутствием консенсуса относительно схемы аннотаций. Значительная часть работ посвящена созданию семантически аннотированных корпусов, например: AMR 1.0 состоит из 13000 предложений [Knight et al., 2014], FrameNet - из 170000 аннотированных предложений [Fillmore, 2003]. Условное семантическое представление фиксирует буквальное значение метафорических предложений. В сочетании с системой идентификации LM такой семантический анализ может служить основой для автоматической интерпретации метафор.

Благодаря усилиям специалистов в области компьютерной лингвистики было успешно разработано несколько подходов к автоматической обработке метафоры: кластеризация имен и глаголов [Shutova, Sun, Korhonen, 2010; Shutova, Sun, 2013], модели векторных пространств [Mohler et al., 2013; Shutova, Van de Cruys, Korhonenm, 2012], веб-поиск с лексико-синтаксическими образцами [Veale, Haо, 2008]. Однако до сих пор эти и другие исследования касались только извлечения текстовых данных для построения метафорических моделей. Принимая во внимание мультимодальный характер метафоры, а также перцептивные свойства концептов и их образность, в качестве наиболее сложной задачи на бли- жайшие годы можно определить разработку таких процедур идентификации метафоры, которые позволили бы использовать не только аннотированные вручную лингвистические ресурсы, но и информацию, полученную на основе данных других модальностей (жестовых, визуальных и др.). Эта задача представляется особенно актуальной для создания репозиториев метафоры в дискурсе, поскольку его целью является раскрытие полного потенциала метафоры в воздействии на мышление людей и манипулировании их мнением.

\section{Заключение}

В представленном исследовании метафор политического дискурса охарактеризованы методологические основы глубинного семантического анализа метафор применительно к созданию двуязычного репозитория метафор с функцией поиска. В статье изложены основные проблемы, стоящие перед лингвистами на начальном этапе разработки репозитория, а также показано, каким образом когнитивный / нейронный взгляд на метафору можно комбинировать с критическим анализом дискурса и теорией культурной концептуализации в рамках одной базы данных.

Промежуточные результаты, полученные на начальном этапе создания репозитория, показывают, что политические метафоры и связанные с ними образные инструменты дискурса отличаются вариативностью, изменчивостью, сценарностью, идеологической и культурной обусловленностью. На примере метафор управления установлено, что степень универсальности и специфичности метафор различна. Английский и русский языки характеризуются общностью большинства способов метафорической концептуализации таких связанных с управлением проблем, как демократия, выборы, преступность и т. д. При этом применение интегративных методик анализа кажущихся универсальными способов концептуализации позволяет выявить некоторые особенности на уровне инференций. Вариативность в использовании метафор связана главным образом со степенью конвенциональности, с набором предпочтительных концептуализаций на уровне сферы-источника, что обусловлено культурным фоном. 
Дальнейшая разработка репозитория на основе каскадной теории позволит определить взаимосвязанные концептуальные сети применительно к политическому дискурсу, представить метафоры на разных уровнях специфичности и сложности, идентифицировать области совпадения метафорических моделей, а также обнаружить культурно маркированные модели концептуализации политических процессов в соответствующих языках.

В ходе работы автора статьи над созданием репозитория в сотрудничестве с коллегами из университета Калифорнии (Беркли) выявлено несколько проблем (как лингвистических, так и связанных с компьютерным моделированием метафоры), которые требуют решения. Если учитывать сценарный характер политических метафор, нельзя не отметить необходимость дальнейшей доработки методов, применяемых для их извлечения и анализа. Поскольку политические метафоры не ограничиваются только вербальной репрезентацией, а находят реализацию в других модальностях (визуальной, жестовой), полученный репозиторий обеспечит возможности построения целостной, мультимодальной, модели метафорического мышления, которая по своим достоинствам превосходит существующие в настоящее время модели, основанные на анализе только языковых данных. Однако такая амбициозная цель требует решения ряда методологически значимых задач, описанных в статье.

\section{СПИСОК ЛИТЕРАТУРЫ}

Болдырев Н. Н., 2013. Интерпретационный потенциал концептуальной метафоры // Когнитивные исследования языка. Вып. 15. С. 12-21.

Будаев Э. В., Чудинов А. П., 2018. Метафора в политической коммуникации. 3-е изд., стер. М. : Флинта : Наука. 249 с.

Banarescu L., Bonial C., Cai S., Georgescu M., Griffitt K., Hermjakob U., Knight K., Koehn. Ph., Palmer M., Schneider N., 2013. Abstract Meaning Representation for Sembanking // Proceedings of the $7^{\text {th }}$ Linguistic Annotation Workshop and Interoperability with Discourse. Sofia, Bulgaria : Association for Computational Linguistics. P. 178-186.

Bogdanova D., 2010. A framework for figurative language detection based on sense differentiation // Proceedings of the ACL 2010 Student Research
Workshop. P. 67-72. URL: https://www.aclweb.org/ anthology/P10-3012.

Burgers C. F., Konijn E. A., Steen J., 2016. Figurative framing: shaping public discourse through metaphor, hyperbole, and irony // Communication Theory. Vol. 26, iss. 4. P. 410-430. DOI: 10.1111/ comt.12096.

Charteris-Black J., 2004. Corpus approaches to critical metaphor analysis. Basingstoke : PalgraveMacMillan. URL: http://eprints.uwe.ac.uk/6739. DOI: $10.1057 / 9780230000612$.

Cienki A., 2008. The application of conceptual metaphor theory to political discourse: Methodological questions and some possible solutions // Political Language and Metaphor: interpreting and changing the world / ed. by T. Carver, J. Pikalo. London ; New York : Routledge. P. 241-256.

Clausner T. C., Croft W., 1999. Domains and image schemas// Cognitive Linguistics. № 10-1. P. 1-32.

Deignan A., 2010. MIP, the corpus and dictionaries: what makes for the best metaphor analysis? // Metaphor and the Social World. Iss. 5.1. P. 145-154. Repository URL: http://eprints.whiterose.ac.uk/ 83351/DOI: 10.1075/msw.5.1.09dei.

Eilts C., Lönneker B., 2002. The Hamburg Metaphor Database // Metaphorik.de. Aus. 3. S. 100-110.

Fass D., 1991. Met*: Amethod for discriminating metonymy and metaphor by computer // Computational Linguistics. Vol. 17, iss. 1. P. 49-90.

Fauconnier G., Turner M., 1998. Conceptual Integration Networks // Cognitive Science. Vol. 22, iss. 2. P. $133-187$.

Feldman J. A., 2006. From Molecule to Metaphor: A Neural Theory of Language. Cambridge, MA : The MIT Press (A Bradford book). $367 \mathrm{p}$.

Fillmore C. J., 1976. Frame semantics and the nature of language // Origins and evolution of language and speech. Annals of the NY Academy of Sciences / ed. by S. R. Harnad, H. D. Steklis, J. Lancaster. New York : Academy of Sciences. Vol. 280. P. 20-32.

Fillmore C. J., 2003. Valency and Semantic Roles: the Concept of Deep Structure Case // Dependency and Valency: An International Handbook of Contemporary Research / ed. by V. Agel [et al.]. Berlin: Walter de Gruyter. P. 457-475.

Gibbs R., 2007. Why cognitive linguists should be concerned with empirical methods // Methods in Cognitive Linguistics / ed. by M. GonzalezMarquez [et al.]. Amsterdam : John Benjamins. P. 2-18. DOI: 10.1075/hcp.18.06gib.

Gibbs R., 2008. The strengths and weaknesses of conceptual metaphor theory: A view from cognitive science // Journal of Foreign Language. Vol. 31. P. 2-12. 
Gibbs R., 2011. Evaluating Conceptual Metaphor Theory // Discourse Processes. Vol. 48, iss. 8. P. 529-562. DOI: 10.1080/0163853X.2011.606103.

Goatly A., 2007. Washing the brain - Metaphor and hidden ideology // Discourse Approaches to Politics, Society and Culture. Vol. 23. DOI: 10.1075/dapsac.23.

Hanks P., 2006. Metaphoricity is gradable // Trends in Linguistic Studies and Monographs. Vol. 17. P. 17-35.

Knight K., Baranescu L., Bonial C., Georgescu M., Griffitt K., Hermjakob U., Marcu D., Palmer M., Schneider N., 2014. Abstract meaning representation (AMR) annotation release 1.0 LDC2014T12 // Web download. Philadelphia : Linguistic Data Consortium. URL: https:// catalog.ldc.upenn.edu/LDC2014T12.

Kövecses Z., 2010. Metaphor: A practical introduction. $2^{\text {nd }}$ ed. Oxford and New York : Oxford University Press. 375 p.

Krennmayr T., 2013. Top-down versus bottom-up approaches to the identification of metaphor in discourse // Metaphorik.de. Aus. 24. P. 7-36.

Lakoff G., 1987. Women, Fire and Dangerous Things: What Categories Reveal about the Mind. Chicago ; London : University Press. 614 p.

LakoffG., 1993. The contemporary theory of metaphor // Metaphor and thought / ed. by A. Ortony. New York : Cambridge University Press. P. 202 251. URL: https://escholarship.org/content/ qt54g7j6zh/qt54g7j6zh.pdf.

Lakoff G., 1994. Master Metaphor List. Berkeley: University of California. URL: http:// cogsci.berkeley.edu.

LakoffG., 1996/2002. Moral politics: How liberals and conservatives think. Chicago, IL : The University of Chicago Press. $471 \mathrm{p}$.

Lakoff G., 2008. The Neural Theory of Metaphor // The Cambridge Handbook of Metaphor and Thought / ed. by W. Raymond, Jr. Gibbs. New York : Cambridge University Press. P. 17-38.

Lakoff G., 2010. Moral politics: How liberals and conservatives think. University of Chicago Press. $471 \mathrm{p}$.

Lakoff G., Johnson M., 1980/2003. Metaphors we live by. Chicago, IL : The University of Chicago Press. $191 \mathrm{p}$.

Langacker R. W., 2008. Cognitive grammar: A basic introduction. Oxford : Oxford University Press. $562 \mathrm{p}$.

Li L., Sporleder C., 2010. Using gaussian mixture models to detect figurative language in context // Human Language Technologies: The 2010 Annual Conference of the North American Chapter of the Association for Computational Linguistics. P. 297-300. URL:
https://www.aclweb.org/anthology/N101039.

Low G., Todd Z., 2010. Good practice in metaphor analysis: guidelines and pitfalls // Metaphor Analysis. Research Practice in Applied Linguistics, Social Sciences and the Humanities / ed. by L. Cameron, R. Maslen. London : Equinox. P. 217-229.

McGlone M. S., 1996. Conceptual Metaphors and Figurative Language Interpretation: Food for Thought? // Journal of Memory and Language. Vol. 35, iss. 4. P. 544-565. DOI: 10.1006/ jmla.1996.0029.

Mohler M., Bracewell D., Tomlinson M., Hinote D., 2013. Semantic signatures for example-based linguistic metaphor detection // Proceedings of the First Workshop on Metaphor in NLP. Atlanta, Georgia. P. 27-35.

Musolff A., 2004. Metaphor and Political Discourse. Analogical Reasoning in Debates about Europe. Basingstoke : Palgrave Macmillan. DOI: 10.1057/ 9780230504516.

Peters W., Wilks Y., 2003. Data-driven detection of figurative language use in electronic language resources // Metaphor and Symbol. Vol. 18, iss. 3. P. 161-173. DOI: 10.1207/S15327868MS1803 03.

Politics, Gender and Conceptual Metaphors, 2009. Politics, Gender and Conceptual Metaphors / ed. by K. Ahrens. Basingstoke : Palgrave Macmillan. 275 p. DOI: 10.1057/9780230245235.

Reddy M. J., 1979. The conduit metaphor - a case of frame conflict in our language about language // Metaphor and thought / ed. by A. Ortony. Cambridge : Cambridge University Press. P. 284-324.

Researching and applying metaphor..., 1999. Researching and applying metaphor (Cambridge Applied Linguistics) / ed. by L. Cameron, G. Low. Cambridge : Cambridge University Press. 312 p. DOI: 1017/ CBO9781139524704.

Ruppenhofer J., Brandes J., 2016. Effect Functors for Opinion Inference // Proceedings of the Tenth International Conference on Language Resources and Evaluation (LREC 2016) / ed. by N. Calzolari [et al.]. Portoroz, Slovenia. P. 2879-2887. URL: https://ids-pub.bsz-bw.de/frontdoor/deliver/ index/docId/5500/file/Ruppenhofer_Brandes_ Effect_Functors_for_Opinion _ Inference 2016.pdf.

Shutova E., 2011. Computational approaches to figurative language. Technical report. University of Cambridge. 217 p. URL: https:// www.cl.cam.ac.uk/techreports/UCAM-CL-TR803.pdf.

Shutova E., Sun L., 2013. Unsupervised metaphor identification using hierarchical graph 
factorization clustering // Proceedings of the 2013 Conference of the North American Chapter of the Association for Computational Linguistics: Human Language Technologies. P. 978-988. URL: https://www.aclweb.org/anthology/N13-1118.

Shutova E., Sun L., Korhonen A., 2010. Metaphor identification using verb and noun clustering // Proceedings of COLING / ed. by C. Huang, D. Jurafsky. P. 1002-1010. URL: https:// www.aclweb.org/anthology/C10-1113.

Shutova E., Van de Cruys T., Korhonenm A., 2012. Unsupervised metaphor paraphrasing using a vector space model // Proceedings of COLING 2012: Posters. P. 1121-1130. URL: https:// www.aclweb.org/anthology/C12-2109.

Skrynnikova I. V., 2016. Cultutral and ideological implications of governance-related metaphors and their translation in media discourse // Proceedings of the International Symposium "Metaphor as means of knowledge communication". Perm : Perm State University. P. 104-108.

Sopory P., Dillard J. P., 2002. The persuasive effects of metaphor:Ameta-analysis //Human Communication Research. Vol. 28, iss. 3. P. 382-419.

Steen G., 2007. Finding Metaphor in Discourse: Pragglejaz and Beyond. Cultura, Lenguaje y Representaciyn // Cultural Studies Journal of Universitat Jaume. Vol. V. P. 9-25.

Steen G. J., Dorst A. G., Herrmann J. B., Kaal A. A., Krennmayr T., Pasma T., 2010a. A Method for Linguistic Metaphor Identification: from MIP to MIPVU. Amsterdam : John Benjamins. xi +238 p. DOI: $10.1075 /$ celcr.14.

Steen G. J., Biernacka E., Dorst A. G., Kaal A. A., LópezRodríguez I., Pasma T., 2010b. Pragglejaz in practice: Finding metaphorically used words in natural discourse // Researching and Applying Metaphor in the Real World / ed. by G. Low [et al.]. Amsterdam and Philadelphia : John Benjamins. P. 165-184.

Stickels E., David O., Dodge E. K., Hong J., 2016. Formalizing contemporary conceptual metaphor theory: A structured repository for metaphor analysis // Constructions and Frames. Vol. 8, iss. 2. P. 166-213. DOI: 10.1075/cf.8.2.03sti.

Veale T., Hao Y., 2008. A fluid knowledge representation for understanding and generating creative metaphors // Proceedings of COLING 2008. P. 945-952. URL: https://www.aclweb.org/ anthology/C08-1119.

Wilkinson P. R., 2002. Thesaurus of traditional English metaphors. London : Routledge. XXVI, 870 p.

Wolff P., Gentner D., 2000. Evidence for role-neutral initial processing of metaphors // Journal of Experimental Psychology: Learning, Memory, and Cognition. Vol. 26, iss. 2. P. 529.

\section{ИСТОЧНИКИ}

Баранов А. Н., Караулов Ю. Н., 1994. Словарь русских политических метафор. М. : Помовский и партнеры. 351 с.

Национальный корпус русского языка. URL: http:// www.ruscorpora.ru.

Treebank. URL: http://treebank.info.

\section{REFERENCES}

Boldyrev N.N., 2013. Interpretatsionnyy potentsial kontseptualnoy metafory [The Interpretative Potential of the Conceptual Metaphor]. Kognitivnye issledovaniya yazyka [Cognitive Studies of Language], iss. 15, pp. 12-21.

Budaev E.V., Chudinov A.P., 2018. Metafora v politicheskoy kommunikatsii [Metaphor in Political Communication]. Moscow, Flinta Publ., Nauka Publ. 249 p.

Banarescu L., Bonial C., Cai S., Georgescu M., Griffitt K., Hermjakob U., Knight K., Koehn. Ph., Palmer M., Schneider N., 2013. Abstract Meaning Representation for Sembanking. Proceedings of the $7^{\text {th }}$ Linguistic Annotation Workshop and Interoperability with Discourse. Sofia, Bulgaria, Association for Computational Linguistics, pp. 178-186.

Bogdanova D., 2010. A Framework for Figurative Language Detection Based on Sense Differentiation. Proceedings of the ACL 2010 Student Research Workshop, pp. 67-72. URL: https://www.aclweb.org/anthology/P10-3012.

Burgers C.F., Konijn E.A., Steen J., 2016. Figurative Framing: Shaping Public Discourse Through Metaphor, Hyperbole, and Irony. Communication Theory, vol. 26, iss. 4, pp. 410-430. DOI: 10.1111/ comt.12096.

Charteris-Black J., 2004. Corpus Approaches to Critical Metaphor Analysis. Basingstoke, PalgraveMacMillan. URL: http://eprints.uwe.ac.uk/6739. DOI: 10.1057/9780230000612.

Cienki A., 2008. The Application of Conceptual Metaphor Theory to Political Discourse: Methodological Questions and Some Possible Solutions. T. Carver, J. Pikalo, eds. Political Language and Metaphor: Interpreting and Changing the World. London, New York, Routledge, pp. 241-256.

Clausner T.C., Croft W., 1999. Domains and Image Schemas. Cognitive Linguistics, no. 10-1, pp. 1-32.

Deignan A., 2010. MIP, the Corpus and Dictionaries: What Makes for the Best Metaphor Analysis? Metaphor and the Social World, iss. 5.1, pp. 145-154. Repository URL: http://eprints.whiterose.ac.uk/ 83351/DOI: 10.1075/msw.5.1.09dei. 
Eilts C., Lönneker B., 2002. The Hamburg Metaphor Database. Metaphorik.de, vol. 3, pp. 100-110.

Fass D., 1991. Met*: A Method for Discriminating Metonymy and Metaphor by Computer. Computational Linguistics, vol. 17, iss. 1, pp. 49-90.

Fauconnier G., Turner M., 1998. Conceptual Integration Networks. Cognitive Science, vol. 22, iss. 2, pp. 133-187.

Feldman J.A., 2006. From Molecule to Metaphor: A Neural Theory of Language. Cambridge, MA, The MIT Press (A Bradford Book). 367 p.

Fillmore C.J., 1976. Frame Semantics and the Nature of Language. S.R. Harnad, H.D. Steklis, J. Lancaster, eds. Origins and Evolution of Language and Speech. Annals of the NY Academy of Sciences. New York, Academy of Sciences, vol. 280, pp. 20-32.

Fillmore C.J., 2003. Valency and Semantic Roles: The Concept of Deep Structure Case. V. Agel, L.M. Eichinger, H.-W. Eroms, P. Hellwig, H.J. Heringer, H. Lobin, eds. Dependency and Valency: An International Handbook of Contemporary Research. Berlin, Walter de Gruyter, pp. 457-475.

Gibbs R., 2007. Why Cognitive Linguists Should be Concerned with Empirical Methods. Methods in Cognitive Linguistics. Amsterdam, John Benjamins, pp. 2-18. DOI: 10.1075/hcp.18.06gib.

Gibbs R., 2008. The Strengths and Weaknesses of Conceptual Metaphor Theory: A View from Cognitive Science. Journal of Foreign Language, vol. 31, pp. 2-12.

Gibbs R., 2011. Evaluating Conceptual Metaphor Theory. Discourse Processes, vol. 48, iss. 8, pp. 529-562. DOI: 10.1080/0163853X.2011.606103.

Goatly A., 2007. Washing the Brain - Metaphor and Hidden Ideology. Discourse Approaches to Politics, Society and Culture, vol. 23. DOI: 10.1075/dapsac.23.

Hanks P., 2006. Metaphoricity is Gradable. Trends in Linguistic Studies and Monographs, vol. 17, pp. 17-35.

Knight K., Baranescu L., Bonial C., Georgescu M., Griffitt K., Hermjakob U., Marcu D., Palmer M., Schneider N., 2014. Abstract Meaning Representation (AMR) Annotation Release 1.0 LDC2014T12. Web Download. Philadelphia, Linguistic Data Consortium. URL: https:// catalog.ldc.upenn.edu/LDC2014T12.

Kövecses Z., 2010. Metaphor: A practical Introduction. Oxford, New York, Oxford University Press. 375 p.

Krennmayr T., 2013. Top-Down Versus Bottom-Up Approaches to the Identification of Metaphor in Discourse. Metaphorik.de, vol. 24, pp. 7-36.
Lakoff G., 1987. Women, Fire and Dangerous Things: What Categories Reveal About the Mind. Chicago, London, University Press. 614 p.

Lakoff G., 1993. The Contemporary Theory of Metaphor. A. Ortony, ed. Metaphor and Thought. New York, Cambridge University Press, pp. 202-251. URL: https://escholarship.org/ content/qt54g7j6zh/qt54g7j6zh.pdf.

Lakoff G., 1994. Master Metaphor List. Berkeley, University of California. URL: http:// cogsci.berkeley.edu.

Lakoff G., 1996/2002. Moral Politics: How Liberals and Conservatives Think. Chicago, IL, The University of Chicago Press. $471 \mathrm{p}$.

Lakoff G., 2008. The Neural Theory of Metaphor. W. Raymond, Jr. Gibbs, eds. The Cambridge Handbook of Metaphor and Thought. New York, Cambridge University Press, pp. 17-38.

Lakoff G., 2010. Moral Politics: How Liberals and Conservatives Think. University of Chicago Press. $471 \mathrm{p}$.

Lakoff G., Johnson M., 1980/2003. Metaphors We Live by. Chicago, IL, The University of Chicago Press. $191 \mathrm{p}$.

Langacker R.W., 2008. Cognitive Grammar: A Basic Introduction. Oxford, Oxford University Press. $562 \mathrm{p}$.

Li L., Sporleder C., 2010. Using Gaussian Mixture Models to Detect Figurative Language in Context. Human Language Technologies: The 2010 Annual Conference of the North American Chapter of the Association for Computational Linguistics, pp. 297-300. URL: https://www.aclweb.org/anthology/N10-1039.

Low G., Todd Z., 2010. Good Practice in Metaphor Analysis: Guidelines and Pitfalls. L. Cameron, R. Maslen, eds. Metaphor Analysis. Research Practice in Applied Linguistics, Social Sciences and the Humanities. London, Equinox, pp. 217-229.

McGlone M.S., 1996. Conceptual Metaphors and Figurative Language Interpretation: Food for Thought? Journal of Memory and Language, vol. 35, iss. 4, pp. 544-565. DOI: $10.1006 /$ jmla.1996.0029.

Mohler M., Bracewell D., Tomlinson M., Hinote D., 2013. Semantic Signatures for Example-Based Linguistic Metaphor Detection. Proceedings of the First Workshop on Metaphor in NLP. Atlanta, Georgia, pp. 27-35.

MusolffA., 2004. Metaphor and Political Discourse. Analogical Reasoning in Debates about Europe. Basingstoke, Palgrave Macmillan. DOI: 10.1057/9780230504516.

Peters W., Wilks Y., 2003. Data-Driven Detection of Figurative Language Use in Electronic Language 
Resources. Metaphor and Symbol, vol. 18, iss. 3, pp. 161-173. DOI: 10.1207/S15327868MS1803_03.

Ahrens K., ed., 2009. Politics, Gender and Conceptual Metaphors. Basingstoke, Palgrave Macmillan. 275 p. DOI: $10.1057 / 9780230245235$.

Reddy M.J., 1979. The Conduit Metaphor - A Case of Frame Conflict in Our Language About Language. A. Ortony, ed. Metaphor and Thought. Cambridge, Cambridge University Press, pp. 284-324.

Cameron L., Low G., eds., 1999. Researching and Applying Metaphor (Cambridge Applied Linguistics). Cambridge, Cambridge University Press. 312 p. DOI: 1017/CBO9781139524704.

Ruppenhofer J., Brandes J., 2016. Effect Functors for Opinion Inference. N. Calzolari, K. Choukri, T. Declerck, M. Grobelnik, B. Maegaard, J. Mariani, A. Moreno, J. Odijk, S. Piperidis, eds. Proceedings of the Tenth International Conference on Language Resources and Evaluation (LREC 2016). Portoroz, Slovenia, pp. 2879-2887. URL: https://ids-pub.bsz-bw.de/frontdoor/deliver/index/ docId/5500/file/Ruppenhofer_Brandes_Effect Functors_for_Opinion_Inference_2016.pdf.

Shutova E., 2011. Computational Approaches to Figurative Language. Technical Report. University of Cambridge. 217 p. URL: https:// www.cl.cam.ac.uk/techreports/UCAM-CL-TR803.pdf.

Shutova E., Sun L., 2013. Unsupervised Metaphor Identification Using Hierarchical Graph Factorization Clustering. Proceedings of the 2013 Conference of the North American Chapter of the Association for Computational Linguistics: Human Language Technologies, pp. 978-988. URL: https://www.aclweb.org/ anthology/N13-1118.

Shutova E., Sun L., Korhonen A., 2010. Metaphor Identification Using Verb and Noun Clustering. C. Huang, D. Jurafsky, eds. Proceedings of COLING, pp. 1002-1010. URL: https:// www.aclweb.org/anthology/C10-1113.

Shutova E., Van de Cruys T., Korhonenm A., 2012. Unsupervised Metaphor Paraphrasing Using a Vector Space Model. Proceedings of COLING 2012: Posters, pp. 1121-1130. URL: https:// www.aclweb.org/anthology/C12-2109.

Skrynnikova I.V., 2016. Cultutral and Ideological Implications of Governance-Related Metaphors and Their Translation in Media Discourse. Proceedings of the International Symposium
"Metaphor as Means of Knowledge Communication”. Perm, Perm State University, pp. 104-108.

Sopory P., Dillard J.P., 2002. The Persuasive Effects of Metaphor: A Meta-Analysis. Human Communication Research, vol. 28, iss. 3, pp. 382-419.

Steen G., 2007. Finding Metaphor in Discourse: Pragglejaz and Beyond. Cultura, Lenguaje y Representaciyn. Cultural Studies Journal of Universitat Jaume, vol. 5, pp. 9-25.

Steen G.J., Dorst A.G., Herrmann J.B., Kaal A.A., Krennmayr T., Pasma T., 2010a. A Method for Linguistic Metaphor Identification: From MIP to MIPVU. Amsterdam, John Benjamins. xi + 238 p. DOI: $10.1075 /$ celcr. 14 .

Steen G.J., Biernacka E., Dorst A.G., Kaal A.A., LópezRodríguez I., Pasma T., 2010b. Pragglejaz in Practice: Finding Metaphorically Used Words in Natural Discourse. Low G., Todd Z., Deignan A., Cameron L., eds. Researching and Applying Metaphor in the Real World. Amsterdam, Philadelphia, John Benjamins, pp. 165-184.

Stickels E., David O., Dodge E.K., Hong J., 2016. Formalizing Contemporary Conceptual Metaphor Theory: A Structured Repository for Metaphor Analysis. Constructions and Frames, vol. 8, iss. 2, pp. 166-213. DOI: 10.1075/cf.8.2.03sti.

Veale T., Hao Y., 2008. A Fluid Knowledge Representation for Understanding and Generating Creative Metaphors. Proceedings of COLING 2008, pp. 945-952. URL: https:// www.aclweb.org/anthology/C08-1119.

Wilkinson P.R., 2002. Thesaurus of Traditional English Metaphors. London, Routledge. XXVI, 870 p.

Wolff P., Gentner D., 2000. Evidence for Role-Neutral Initial Processing of Metaphors. Journal of Experimental Psychology: Learning, Memory, and Cognition, vol. 26, iss. 2, p. 529.

\section{SOURCES}

Baranov A.N., Karaulov Yu.N., 1994. Slovar russkikh politicheskikh metaphor [Dictionary of Russian Political Metaphors]. Moscow, Pomovskiy i partner Publ. $351 \mathrm{p}$.

Natsionalnyy korpus russkogo yazyka [National Corpus of the Russian Language]. URL: http:// www.ruscorpora.ru.

Treebank. URL: http://treebank.info. 


\section{Information about the Author}

Inna V. Skrynnikova, Candidate of Sciences (Philology), Associate Professor, Germanic and Romance Philology Department, Volgograd State University, Prosp. Universitetsky, 100, 400062 Volgograd, Russia, i.skrynnikova@volsu.ru, https://orcid.org/0000-0002-2390-7866

\section{Информация об авторе}

Инна Валериевна Скрынникова, кандидат филологических наук, доцент кафедры германской и романской филологии, Волгоградский государственный университет, просп. Университетский, 100, 400062 г. Волгоград, Россия, i.skrynnikova@volsu.ru, https://orcid.org/0000-0002-2390-7866 